\title{
Der Untersuchungsteil Motorik im Pretest des Kinder- und Jugendgesundheitssurveys
}

H. Kahl

J. Emmel

\author{
The Examination of Motor Activity in the Pilot Study of the National Health \\ Interview and Examination Survey for Children and Adolescents
}

\section{Zusammenfassung}

Im Kinder- und Jugendgesundheitssurvey ist die Motorik ein Untersuchungsbereich zur physischen Gesundheit. Das unterstreicht den Stellenwert der Bewegung in ihrem Einfluss auf die altersgemäße Entwicklung. In dieser erstmalig für die Bundesrepublik repräsentativen Studie sollen Daten zum motorischen Entwicklungsstand der Kinder und Jugendlichen gewonnen werden, um Grundlagen für den Einsatz gezielter Interventionsmaßnahmen zu schaffen. Unter Surveybedingungen müssen sich Umfang und Auswahl motorischer Tests auf ein Minimalprogramm beschränken, nämlich auf Prüfungen zur Koordination, Haltung, Beweglichkeit und Kraftausdauer. In einem Pretest wurden Eignung, Durchführbarkeit und Aussagekraft dieser Einzeltests überprüft und die methodischen Schwierigkeiten aufgezeigt, die die Standardisierbarkeit der Tests und die Anforderungen an das Untersuchungspersonal betreffen. Ein weiteres Anliegen war, den Altersgang und die Geschlechtsspezifik in der motorischen Entwicklung durch die eingesetzten Tests abbilden zu können. Für den bundesweiten Kinder- und Jugendgesundheitssurvey können folgende Prüfungen empfohlen werden: Koordination (Rückwärtsbalancieren, Einbeinstand, seitliches Hin- und Herspringen), Kraftausdauer (Sit up, Liegestütz) und Beweglichkeit (Rumpfvorbeuge).

\section{Schlüsselwörter}

Kinder und Jugendliche · Survey · motorische Tests · Standardisierung

\begin{abstract}
In the Health Survey for Children and Adolescents the examination of motor activity is one aspect of physical health covered by the study. This underlines the importance of physical activity for physical development in early years. This first representative child and adolescent study for Germany intends to obtain data on motor activity and to allow for the implementation of specific intervention programmes encouraging physical activity. The specific general conditions under which the survey is conducted restrict the selection and scope of possible instruments to a minimal programme, including fitness tests, strength in combination with endurance and coordinative skills as well as flexibility. In a pilot study the suitability, feasibility and the obtained evidence of selected single motor tests were tested. This article explains the choice of instruments and methods used in the examination of physical fitness. It also discusses methodological difficulties which affect the standardisation of tests and the requirements regarding personnel. A major concern of the pilot study was the evaluation of tested instruments with regard to gender and age differences. For the main survey the following tests are recommended: coordination (balancing backwards, one-leg-footing, sideway jumping), perseverance (sit-ups, push-ups), and flexibility (trunk bending).
\end{abstract}

Key words

Children and adolescents $\cdot$ survey $\cdot$ motor tests $\cdot$ standardisation 


\section{Einführung}

In den letzten Jahrzehnten hat die Technisierung der Umwelt und die zunehmende Nutzung neuer Medien auch bei Kindern zu einem veränderten Freizeitverhalten geführt, das mit deutlichen Einschränkungen ihrer Bewegungserfahrungen im Alltag verbunden ist. Folgen dieses Bewegungsmangels zeigen sich in der Zunahme von Haltungs- und Koordinationsstörungen und einer abnehmenden Ausdauer [Brandt et al. 1997, Kleine 1997, Weineck et al. 1997, Dordel 1998, Klaes et al. 2001]. Durch die fehlende Konsistenz der Daten aus den vorhandenen Einzelstudien ist eine bevölkerungsbezogene Aussage zur altersgemäßen motorischen Entwicklung von Kindern und Jugendlichen nur eingeschränkt möglich [Gaschler 2001].

Die Aufnahme des Untersuchungsteils Motorik in den Kinder- und Jugendgesundheitssurvey [Kurth et al. 2002] unterstreicht den Stellenwert der körperlichen Aktivität als Einflussfaktor auf das Entwicklungsgeschehen und auf den Gesundheitszustand im Kindesalter. Auch im Hinblick auf mögliche Krankheitsfolgen im Erwachsenenalter ist das Wissen um das Ausmaß der körperlichen Inaktivität im Kindes- und Jugendalter von großer Bedeutung [Daugs et al. 1996, Sönnichsen et al. 1997, Zieske et al. 2002].

Der Pretest, in dem 1630 Kinder und Jugendliche im Alter von 0 bis 17 Jahren untersucht wurden, sollte die Eignung und Durchführbarkeit ausgewählter Methoden unter den Bedingungen eines Surveys prüfen [Kamtsiuris et al. 2002]. Das umfasste die Einschätzung der Testdurchführung unter methodischen Aspekten der Standardisierung ebenso wie die Einschätzung der räumlichen, zeitlichen und personellen Voraussetzungen im Survey und die Erklärungen zur Akzeptanz der Untersuchung bei Kindern und Eltern. Die ausgewählten Einzeltests sollten Aussagen in Bezug auf den Altersgang und die Geschlechtsspezifik ermöglichen.

\section{Bedingungen für die Testauswahl}

Die spezifischen Rahmenbedingungen des Surveys, wie die zeitliche Vorgabe von ca. 15 Minuten für den Motorikteil und die Situation mit einem oft beengten Untersuchungsraum, schränkten die Auswahl der motorischen Übungen von vornherein auf Tests ein, die wenig Zeit in Anspruch nehmen und keinen großen Aktionsradius erfordern wie Sprung-, Wurf- oder Lauftests. Verletzungsgefahren mussten ausgeschaltet werden. Von Bedeutung war ebenfalls, dass die Tests, nach entsprechender Schulung, von einer Kinderkrankenschwester abgenommen werden können.

Das methodische Grundproblem bestand darin, motorische Prüfbereiche zu definieren, die wesentliche koordinative und konditionelle Fähigkeitsbereiche mit hoher gesundheitlicher Relevanz erfassen. Konkrete Schwierigkeiten gab es, aus dem verfügbaren Untersuchungsinventar im Motorik-Sportbereich solche motorischen Einzelübungen zu finden, die auch nach Herauslösung aus validierten Testbatterien die ausgewählten Prüfbereiche kindlicher Motorik ausreichend erfassen und die alters- und geschlechtsspezifische Entwicklung widerspiegeln. Die Festlegung der Testaufgaben erfolgte in Abstimmung mit externen Experten.
Neben den methodischen Aspekten muss bei der Auswahl der Tests auch der Gerätebedarf Berücksichtigung finden. Leichter Transport und hohe Stabilität der Geräte spielen bei häufigem Wechsel der Untersuchungsorte durchaus eine Rolle.

\section{Testspektrum}

Bei der Auswahl von Tests zur Bestimmung von vorwiegend koordinativen Fähigkeiten waren der für das Vorschulalter entwickelte Test MOT 4-6 [Zimmer, Volkamer 1984] und der Körperkoordinationstest für Kinder (KTK) [Kiphard, Schilling 1974] für die Altersgruppe 5 bis 14 Jahre als vollständige Verfahren aufgrund der Testkonstruktion, der jeweiligen Zielstellung, der Zeitdauer und der Anforderungen an die Durchführung für den Survey nicht praktikabel. Als konditionelle Übungen, die unter Surveybedingungen machbar erschienen, wurden Tests für die Überprüfung der Kraftausdauer von Schulter-/Arm-, Rückenund Bauchmuskulatur ausgewählt. Ein Test zur Haltungsbeurteilung und zur Beweglichkeit vervollständigten das Testspektrum [Beck, Bös 1995]. Im Pretest wurden damit aus vier motorischen Prüfbereichen Tests erprobt:

- Koordination: Vorwärts- und Rückwärtsbalancieren, monopedales Hüpfen, Tennisring fangen, mit den Zehen greifen, seitliches Hin- und Herhüpfen, Einbeinstand

- Haltung: Matthiass-Test

- Beweglichkeit: Rumpfvorbeuge

- Kraftausdauer: Sit up und Liegestütz.

Für den Motorikteil wurden eine Sportmatte, ein Balken für den Einbeinstand, eine Sportbank, ein Messstab zur Quantifizierung der Rumpfvorbeuge und eine Stoppuhr benötigt.

In einer Zwischenbilanz nach der ersten Pretestphase wurden Fragen der Durchführbarkeit, der Bewertung und der Aussagefähigkeit der Testaufgaben diskutiert und für einzelne Altersgruppen Umstellungen im Methodenspektrum (Tab.1) vorgenommen, die sowohl Streichungen als auch Erweiterungen von Testaufgaben beinhalteten.

\section{Methodische Erprobung}

\section{Altersgruppe 4 und 5 Jahre}

Für die 4- bis 5-Jährigen haben sich die schon in der Literatur beschriebenen Schwierigkeiten der Testdurchführung bestätigt. Die fremde Umgebung und die Stimmungslage entscheiden häufig über die Bereitschaft der Kinder, die demonstrierten Untersuchungen selbst durchzuführen. Die Probanden dieser Altersgruppe brauchten oft viel Zuspruch, um ihre Hemmungen zu überwinden. Eine einmalige Erklärung und Vorgabe der Übung reichte oft nicht aus und die standardisierte Durchführung wurde schwierig, weil ein sehr individuelles Eingehen auf die kleinen Probanden nötig war. Die Stichprobe umfasste 27 Kinder in der Altersgruppe 4 Jahre und 35 Kinder in der Altersgruppe 5 Jahre und erlaubt trotz der genannten Probleme sowohl eine Einschätzung zur Durchführbarkeit als auch zur Aussagefähigkeit der erprobten Testaufgaben. 
Tab. 1 Untersuchungsspektrum der Motorik im Pretest

\begin{tabular}{|c|c|c|c|c|c|}
\hline \multicolumn{2}{|c|}{ Altersgruppe 4-5 Jahre } & \multicolumn{2}{|c|}{ Altersgruppe 6-10 Jahre } & \multicolumn{2}{|c|}{ Altersgruppe 11-17 Jahre } \\
\hline $\begin{array}{l}\text { Pretest } \\
\text { 1. Hälfte }\end{array}$ & $\begin{array}{l}\text { Pretest } \\
\text { 2. Hälfte }\end{array}$ & $\begin{array}{l}\text { Pretest } \\
\text { 1. Hälfte }\end{array}$ & $\begin{array}{l}\text { Pretest } \\
\text { 2. Hälfte }\end{array}$ & $\begin{array}{l}\text { Pretest } \\
\text { 1. Hälfte }\end{array}$ & $\begin{array}{l}\text { Pretest } \\
\text { 2. Hälfte }\end{array}$ \\
\hline \multicolumn{2}{|c|}{ balancieren (vorwärts, rückwärts)) } & Matthiass-Test & gestrichen & Matthiass-Test & gestrichen \\
\hline \multirow[t]{6}{*}{ monopedales Hüpfen } & mit den Zehen greifen & mit den Zehen greifen & gestrichen & & \\
\hline & Tennisring fangen & seitlich Hin- $\iota$ & pringen & & \\
\hline & & Rumpf & & & \\
\hline & & Einbe & & Einbein & iene \\
\hline & & & & & \\
\hline & & & Liegestütz & & \\
\hline
\end{tabular}

Balancieren vorwärts und rückwärts (aus MOT 4-6): Bei dieser Übung wird die Anzahl der Abweichungen von einer markierten, $2 \mathrm{~m}$ langen Linie bei selbst bestimmter Schrittlänge erfasst. Den Kindern fällt es generell leichter, vorwärts als rückwärts zu balancieren, wobei dem Rückwärtsbalancieren die größere Aussagekraft für den Prüfbereich Koordination mit Präzision zuzumessen ist. Bei den 4-jährigen Probanden differenziert dieser Test ausreichend, bei den 5-jährigen nimmt die Anzahl der Abweichungen beim Rückwärtsbalancieren deutlich ab. Mädchen in diesem Alter zeigen eine bessere Koordination als Jungen. Der Test Rückwärtsbalancieren zeigt einen Entwicklungszuwachs an und kann Aussagen zum Stand der motorischen Entwicklung im Bereich Koordination liefern. Als alleinige Testaussage für die Erfassung der koordinativen Fähigkeiten in dieser Altersgruppe ist er nicht ausreichend.

Monopedales Hüpfen: Die Mehrzahl der Kinder bewältigte das Hüpfen auf einem Bein (5-mal) auf dem selbst gewählten Sprungbein ohne Schwierigkeiten. In aller Regel wird das rechte Bein als Sprungbein vorgezogen. Das Erkennen koordinativer Auffälligkeiten hat sich mit diesem Einzeltest nicht bewährt. Er wurde nach Ablauf der ersten Pretestphase nicht mehr durchgeführt.

Tennisring fangen (aus MOT 4-6): Diese in der zweiten Pretestphase neu aufgenommene Übung zur Prüfung der Auge-HandKoordination und Reaktionsschnelligkeit hat sich ebenfalls nicht bewährt. Die Räumlichkeiten schränkten die Testdurchführung mit der vorgeschriebenen Fangstrecke von 4 m ein.

Mit den Zehen greifen (aus MOT 4-6): Diese Probe wurde erst in der zweiten Pretesthälfte für die 4- bis 5-Jährigen übernommen, da er von 6- bis 10-jährigen Kindern in der Mehrzahl geschafft wurde. Als Test zur Überprüfung des Standgleichgewichtes und der Auge-Fuß-Koordination ist er für diese Altersgruppe aussagefähig.

Im Pretest hat sich bestätigt, dass es schwierig ist, aus dem vorhandenen Methodeninventar für das Vorschulalter (z.B. MOT 4-6) geeignete, aussagefähige Einzeltests für den Einsatz in Surveys zu finden. Ein aussagefähiges Screening-Instrument zur Erfassung motorischer Auffälligkeiten in diesem Altersbereich zu entwickeln bleibt weiterhin eine Herausforderung für die sportwissenschaftliche Forschung.

\section{Altersgruppe 6 bis 10 Jahre}

Die Durchführung der Testaufgaben bereitete in dieser Altersgruppe keine Schwierigkeiten. Bereitschaft, Verständnis und Leistungsmotivation waren bei allen Kindern gegeben, die ein großes Interesse hatten, ihre körperliche Leistungsfähigkeit zu zeigen.

Seitliches Hin- und Herspringen (aus KTK): Das Überspringen einer Trennlinie mit beiden Beinen und das gleichzeitige laute Zählen der geschafften Sprünge bereitete allen Kindern Freude. Die Ergebnisse zeigen für Jungen und Mädchen einen altersgemäßen Entwicklungszuwachs in der Leistung, d. h., mit zunehmendem Alter steigt auch die Anzahl der Sprünge. Dabei gibt es kaum Unterschiede zwischen den Mittelwerten bei Jungen und Mädchen. Diese geringe Geschlechtsspezifik zeigte sich bereits für das seitliche Hin- und Herspringen in der Normierungsstichprobe des Körperkoordinationstests [Kiphard, Schilling 1974]. Für die Prüfbereiche Koordination unter Zeitdruck sowie Kraftausdauer der Beinmuskulatur dokumentiert dieser Test deutlich den altersspezifischen Entwicklungszuwachs für 6- bis 10-Jährige. Er ist für den Surveyeinsatz gut geeignet.

Einbeinstand: Das Stehen auf einem Bein (nach Wahl) über 15 Sekunden wurde in den Altersgruppen 6 bis 10 Jahre differenziert erhoben. Mit zunehmendem Alter steigt diese Fähigkeit, wobei

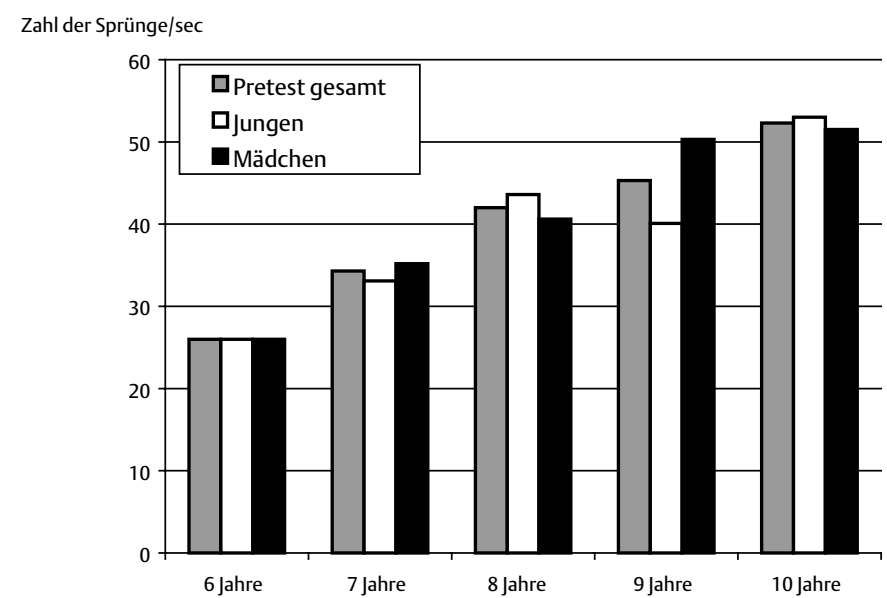

Abb. 1 Seitliches Hin- und Herspringen nach Alter und Geschlecht (Mittelwerte). 
ca. 20\% der 7-jährigen Mädchen und Jungen die Leistung von 15 Sekunden nicht erreichten. Zwischen den 7-Jährigen und 8-Jährigen kommt es allerdings zu einem Leistungsabfall, der darauf zurückzuführen ist, dass die Kinder der Altersgruppe ab 8 Jahre die Augen beim Einbeinstand geschlossen halten mussten. Um die unterschiedlichen Leistungsanforderungen bei dieser Übung zu umgehen, soll der Einbeinstand im Hauptsurvey auch bei den jüngeren Altersgruppen einheitlich auf dem Balken durchgeführt werden [Bös et al. 2001].

Liegestütz: Die Aufnahme des Tests Liegestütz in diese Altersgruppe ist damit begründet, dass der Sit up als alleiniger Test für die Kraftausdauer die muskuläre Leistungsfähigkeit (vorwiegend Bauchmuskulatur) zu einseitig abbildet. Mit dem Liegestütz wird auch der Teilbereich obere Extremitäten (schulterblattfixierende Muskulatur) erfasst, wobei Hinweise auf Haltungsinsuffizienzen aufgrund muskulärer Schwächen erkannt werden.

Matthiass-Test: Im Pretest bestätigte sich, dass die Beurteilung der Halteleistung, des Haltungsgefühls und der Haltungskoordination durch den Matthiass-Test mit einer hohen Subjektivität verbunden ist. Der Test setzt die Begutachtung durch einen versierten Untersucher voraus, der zwischen tiefer Ruhehaltung und habitueller Haltung sowie der vollen Aufrichtung differenzieren und somit eine normale Haltungsschwäche Grad I und II unterscheiden kann. Eine Alternative zu der stark subjektiv geprägten Begutachtung der Haltung und der Rückenformen durch den Matthiass-Test bietet unter Umständen das sog. Orthotronic-Triflexometer. Das elektromechanische Messgerät erfasst nicht-invasiv die äußere Wirbelsäulenkontur in unbeeinflusster Haltung und im aufrechten Stand.

\section{Altersgruppe 6 bis 17 Jahre}

Die Durchführung der drei Testaufgaben Sit up, Einbeinstand und Rumpfvorbeuge über den gesamten Altersbereich und über beide Pretestphasen hat viele Hinweise zur Optimierung der Testdurchführung geliefert und durch die größere Stichprobe die Aussagen zum Altersgang und zur Geschlechtsdifferenzierung verdeutlicht. Das gilt ebenso für den Liegestütz, der ab der zweiten Pretesthälfte auch für die 6- bis 10-jährigen Kinder in das Testprogramm aufgenommen wurde.
Sit up: Im Ergebnis dieser Übung zur Beurteilung der Bauchmuskelkraft bestätigten sich Unterschiede zwischen den Geschlechtern und den Altersgruppen. Vom sechsten bis zehnten Lebensjahr ist der Entwicklungszuwachs sowohl bei Mädchen als auch bei Jungen ausgeprägt. Ab dem zehnten Lebensjahr nehmen durch die Stagnation der Leistungen bei den Mädchen die Geschlechtsdifferenzen noch zu (Abb. 2).

Einbeinstand: Bei diesem Koordinationstest mussten die Kinder ab 11 Jahre mit einem Bein auf einer T-Schiene stehen und das Gleichgewicht halten, wobei die Anzahl der Bodenberührungen des Spielbeines während einer Minute erfasst wurde. Insgesamt schien die Aufgabe für alle Altersgruppen nicht einfach zu sein, zumal in der ersten Pretesthälfte dieser Test barfuß bzw. auf Strümpfen durchgeführt wurde. Zum Teil benötigten die Kinder und Jugendlichen mehr als zehn Versuche in allen Altersgruppen. Es gibt nur geringe Geschlechtsunterschiede. Im Vergleich zur Normstichprobe von [Beck und Bös 1995] ist zu sehen, dass prinzipiell das Leistungsspektrum von weit unterdurchschnittlich bis weit überdurchschnittlich innerhalb der Altersgruppen vertreten ist. Das spricht für eine gute Differenzierungsfähigkeit des Tests. Aufgrund von Beschwerden der Kinder über Schmerzen an den Fußsohlen beim Balancieren wurde in der zweiten Pretesthälfte der Einbeinstand mit Schuhen erlaubt. Da unterschiedliches Schuhwerk aber durchaus Einfluss auf das Testergebnis haben kann, soll im Hauptsurvey vorgegeben werden, Sportschuhe mitzubringen.

Die weiteren Tests Rumpfvorbeuge und Liegestütz zeigen ebenfalls durchschnittliche Zunahmen im Altersgang für beide Geschlechter. Mädchen zeigen dabei eine größere Beweglichkeit bei der Rumpfvorbeuge, Jungen eine höhere Kraftausdauer beim Liegestütz.

\section{Optimierung der Testdurchführung}

Die Qualitätskontrollen vor Ort und die Erfahrungen der Untersucherin haben in Abstimmung mit den Kooperationspartnern neben Veränderungen im Methodenspektrum auch eine Reihe von Hinweisen zur besseren Standardisierung der Testdurchführung und der Gestaltung von Untersuchungsbedingungen ergeben. Sie umfasst:

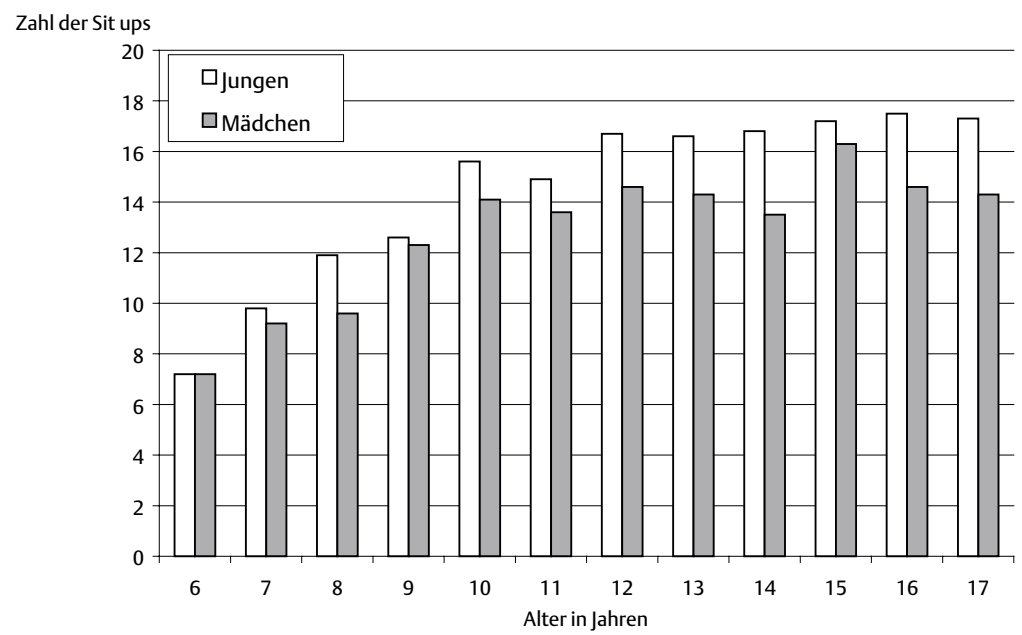

Abb. 2 Sit ups nach Alter und Geschlecht (Mittelwerte). 
- die Gestaltung der Übungsabfolge derart, dass eine geringe Vordehnung der Muskulatur für nachfolgende Übungen erreicht wird. Dazu sind die Übungen mit koordinativen Schwerpunkten an den Anfang gesetzt. Zwischen Sit up und Liegestütz soll den Teilnehmern eine Pause von längstens einer Minute zur Erholung gewährt werden;

- die Veranschaulichung der Bewegungsabläufe für die Kinder bei den Übungen Sit up und Liegestütz in Form von Fotos mit einzelnen charakteristischen Körperstellungen;

- die Präzisierung der Testinstruktionen;

- das Minimieren der Untersuchervariabilität durch den Einsatz möglichst motometrischer Methoden;

- die Erinnerung an das Mitbringen einer sportgeeigneten Kleidung bei der Terminvergabe zur Untersuchung (eng anliegende Kleidung schränkt den Bewegungsradius ein, weite Kleidungsstücke erlauben hingegen keine genaue Bewertung der Bewegungsausführung).

\section{Akzeptanz durch Kinder und Eltern}

Grundsätzlich wurden die Motoriktests von allen Probanden angenommen. Es gab während des gesamten Pretests drei Probanden, die eine Motorikübung ablehnten. Die Motivation, eine höchstmögliche Leistung im sportlichen Teil der Studie anzustreben, war je nach Untersuchungsort sehr unterschiedlich: Probanden, die in der Schule befragt wurden, waren oft durch Mitschüler genau über den Übungsablauf informiert und die Ergebnisse der Tests wurden ausgetauscht, was zu einer Wettbewerbssituation führte. Die Motivation, Leistung zu bringen, war hier sehr hoch. Bei den Prüfungen in einem Untersuchungszentrum erfordert die Durchführung der motorischen Übungen besonders bei den 4 - bis 5-jährigen Kindern ein individuelles Eingehen. In der Altersgruppe 6 bis 10 Jahre gab es die größte Teilnahmebereitschaft, während jugendliche Probanden, die nur auf Drängen der Eltern mit zur Untersuchung gekommen waren, zwar bereit waren, den Motorikteil zu absolvieren, aber trotz Aufforderung keine große Leistungsmotivation aufbrachten. Für Eltern, die an Hintergrundinformationen zu den Tests interessiert sind, sollten dem Untersucher Argumentationshilfen zur Hand gegeben werden.

\section{Schlussfolgerungen}

Im Pretest hat sich gezeigt, dass die Mehrzahl der ausgewählten motorischen Einzeltests für die Altersgruppen ab 6 Jahre unter den Bedingungen des Surveys einsetzbar ist. Die Ergebnisse der Tests differenzieren nach Alter und Geschlecht und können bei entsprechender Stichprobengröße, anhand der Mittelwerte bzw. von Verteilungen, das motorische Entwicklungsniveau bzw. die muskuläre Leistungsfähigkeit ausgewählter Muskelbereiche für die entsprechenden Altersgruppen beschreiben. Um die Vergleichbarkeit und Verallgemeinerungsfähigkeit der Testergebnisse zu gewährleisten, müssen diese unter den festgelegten
Standards durchgeführt werden. Räumliche, zeitliche und personelle Voraussetzungen müssen einem Mindeststandard genügen. Da auch die Motivation der Teilnehmer einen großen Einfluss auf das Leistungsergebnis nimmt, sollten gleiche äußere Untersuchungsbedingungen gewährleistet sein.

Durch die Möglichkeit, in einem dem Survey angegliederten Modul das Methodenspektrum komplementär zu untersuchen, wurde für die Hauptphase des Kinder- und Jugendgesundheitssurveys festgelegt, sich im Kernsurvey nur auf zwei relevante motorische Prüfbereiche zu konzentrieren: In der Altersgruppe 4 bis 10 Jahre soll die Koordination umfassender untersucht werden und in der Altersgruppe 11 bis 17 Jahre die Ausdauerleistungsfähigkeit [Bös et al. 2002].

\section{Literatur}

${ }^{1}$ Beck J, Bös K. Normwerte motorischer Leistungsfähigkeit. Bundesinstitut für Sportwissenschaft. In: Sport und Buch Srauss (Hrsg).. Köln: Sport 1995

${ }^{2}$ Bös K, Opper E, Woll A et al. Das Karlsruher Testsystem für Kinder (KATS-K) - Testmanual. Haltung und Bewegung 2001; 4: 4-66

3 Bös K, Heel J, Romahn N et al. Untersuchungen zur Motorik im Rahmen des Kinder- und Jugendgesundheitssurveys. Gesundheitswesen 2002; 64 (Sonderheft 1): 80-87

${ }^{4}$ Brandt K, Eggert D, Jendritzki $\mathrm{H}$ et al. Untersuchungen zur motorischen Entwicklung von Kindern im Grundschulalter in den Jahren 1985 und 1995. Motorik 1997; 22: 101-107

${ }^{5}$ Daugs R, Blischke K, Marschall F et al. Kognition und Motorik. Hamburg 1996

${ }^{6}$ Dordel S. Ätiologie und Symptomatik motorischer Defizite und Auffälligkeiten. In: Bundeszentrale für gesundheitliche Aufklärung (BZgA) (Hrsg). Gesundheit von Kindern - Epidemiologische Grundlagen. Köln: Expertenworkshop 1996 1998; 98-111

${ }^{7}$ Gaschler P. Motorik von Kindern und Jugendlichen heute - Eine Generation von „Weicheiern, Schlaffis und Desinteressierten?“ (Teil 3). Haltung und Bewegung 2001; 1: 5-17

${ }^{8}$ Kamtsiuris P, Bergmann KE, Dippelhofer A et al. Der Pretest des Kinder- und Jugendgesundheitssurveys: Methodische Aspekte und Durchführung. Gesundheitswesen 2002; 64 (Sonderheft 1): 99-106

${ }^{9}$ Kiphard EJ, Schilling F. Körperkoordinationstest für Kinder. KTK. Manual von Schilling F. Belts. Weinheim: Test Gesellschaft 1974

${ }^{10}$ Klaes L, Rommel A, Cosler D et al. WIAD-Studie: Bewegungsstatus von Kindern und Jugendlichen in Deutschland. Imquadrat multimedia gmbh, ISBN 3-89 152-086-7. Frankfurt am Main 2001

11 Kleine W. Entwöhnen wir unseren Kindern die Bewegung? Sportunterricht 1997; 46: 487-493

12 Kurth BM, Bergmann KE, Hölling $\mathrm{H}$ et al. Der bundesweite Kinder- und Jugendgesundheitssurvey - Das Gesamtkonzept. Gesundheitswesen 2002; 64 (Sonderheft 1): 3-11

13 Sönnichsen C, Weineck J, Köstermeyer G. PEP - eine Studie zur Präventionserziehung - Teil 3: Motorisches Leistungsvermögen und kardiovasculäres Risiko von Schulanfängern. Haltung und Bewegung 1997; 3. $5-10$

14 Weineck J, Köstermeyer G, Sönnichsen C. PEP eine Studie zur Präventionserziehung - Teil 1: Zum motorischen Leistungsvermögen von Schulanfängern. Haltung und Bewegung 1997; 17: 5-16

15 Zieske AW, Malcolm DT, Strong JP. Natural history and risk factors of arteriosclerosis in children and youth: The PDAY study. Pediatric Pathology and Molecular Medicine 2002; 21: 213-237

16 Zimmer R, Volkamer M. MOT4-6 - Motoriktest für vier- bis sechsjährige Kinder. Manual. Weinheim: Belts Test Gesellschaft 1984 